\title{
Experimental Setup for the Analysis of Vortices
}

\author{
S. Nizetic ${ }^{\dagger}$ F. Grubisic-Cabo and M. Bugarin \\ Faculty of Electrical Engineering, Mechanical Engineering and Naval Architecture, \\ University of Split, Split, 21000, Croatia \\ $\dagger$ Corresponding Author Email: snizetic@fesb.hr
}

(Received December 19, 2013; accepted February 5, 2014)

\begin{abstract}
This paper presents a specific experimental setup in which artificial vortices are successfully created and maintained under controlled conditions. The developed vortex simulation chamber is demonstrated to be crucial to the study of vortices and to improve the understanding of vortex nature and behaviour. Furthermore, the general simulation chamber characteristics, constructional details and working principle are elaborated in detail. Preliminary experimental results are also discussed. Specifically, the influence of the water vapour content on the pressure potential and the vortex stability has been analysed. The specific friction work in the vortex system has also been analysed; the novel specific vortex efficiency factor has been introduced. The gained research results and conclusions are important to understand the complex nature of vortex systems, which can be useful for both meteorological purposes and energy concepts research, where convective vortices are assumed to be used as heat engines.
\end{abstract}

Keywords: vortices, experimental setup, pressure potential, specific vortex efficiency.

\section{NOMENCLATURE}

$c_{p} \quad$ specific heat capacity of air,

$e_{k} \quad$ specific kinetic energy

$e_{f} \quad$ specific friction energy

$l_{v} \quad$ latent heat of vaporization

$p \quad$ pressure

$v \quad$ radial inflow air velocity

$\varepsilon \quad$ specific vortex efficiency factor

$w \quad$ specific work
$W_{i r r}^{a b} \quad$ specific friction work in nonreversible

processes

$\rho \quad$ density of air

$\eta \quad$ thermodynamic vortex efficiency

$\gamma \quad$ ratio between friction and overall work

$\Delta T_{n a} \quad$ temperature perturbation

$\Delta q \quad$ specific absolute humidity difference

\section{INTRODUCTION}

It is well-known that natural convective vortices, such as dust devils, tornadoes, and waterspouts, can cause hazards and economic problems in specific geographical locations. A large amount of funds and resources have been devoted to providing field observations and to conducting research in this specific field to reduce unwanted impact on society. Specifically, understanding the behaviour vortices and discovering the general features of their influential physical parameters is important for convective vortices systems. Therefore, theoretical research in this field is necessary, but experimental approaches, especially field experimental approaches, are also a crucial, Wurman et al. (2013), Palmer et al. (2011), Inoue et al. (2011), Chandrasekar et al. (2010), Bech et al. (2009), Renno (2004), Metzger et al. (2002), Bluestein and Pazmany (2000), Mattsson et al. (1993) and Hess and Spillane (1990). Dessens (1962) published a paper entitled "Man-made tornadoes", in which he elaborated an experimental plant, Meteotron, in detail. The Meteotron experiment was successfully performed (artificial whirlwinds were simultaneously produced), and this study was the first large-scale experiment involving vortices. Michaud (1975) proposed an intriguing idea for the controlled use of convective vortices to produce electricity. Michaud's idea was important because it was the first proposed technical concept in which convective vortices are assumed to be used as heat engines that can produce useful work (for example, from industrial waste heat as an available heat source, which increases the overall efficiency of conventional power plants). Later, Michaud developed his idea as a business plan, and a large-scale prototype plant is expected to be operational Michaud (2013) in the future. The theoretical and experimental research of convective vortices can be divided in the two approaches. The first approach addresses the meteorological aspect of natural convective vortices, Renno and Ingersoll (1996), Renno and Bluestein (2001), Renno et al. (1998, 2004), Emanuel (1989), 


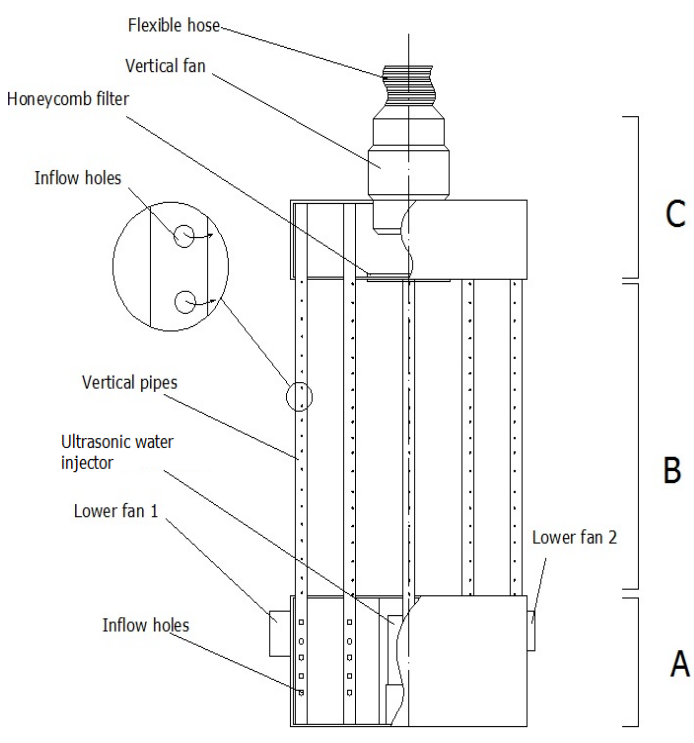

Fig. 1.a Simplified schematic overview of the simulation chamber

Natarajan and Hangan (2012), Fred et al. (2008) such as their behaviour, prediction, vortex genesis, etc. The second approach relates to the analysis of the possible implementation of convective vortices as heat engines Michaud (1995,1996,1999), Ninic and Nizetic (2009), Nizetic (2010), Ninic et al. (2006), Nizetic, (2010, 2011) to establish new energy sources. However, both previously mentioned approaches are useful to generate knowledge and experience that would improve the understanding of complex physical processes in convective vortex systems Hasegawa et.al. 2008, Mukunda et.al.2009, Firouzabadi et.al. 2010, Sobolik et.al 2011 and Paul et. al. 2011.

The objective of this paper was to elaborate a specific developed experimental setup of a convective vortex simulation chamber, as well as to present and discuss preliminary research results that will be useful for further research on this specific research topic.

\section{VORTEX SIMULATION CHAMBER DESIGN}

To research the characteristics and behaviour of vortices in general, a simulation was built according to the design that was proposed by Grazulis (1998). The simulation chamber of the original Grazulis design was slightly redesigned to expand chamber applications, i.e., to ensure the establishment of planned research tasks. According to Fig. 1., the simulation chamber consists of three main parts, i.e. the lower - A, central - B and the upper - C part. The lower part of the chamber - $\mathrm{A}$ is a double box, the central section of which contains an ultrasonic water injector to ensure vortex visibility. A perforated plate, which contained drilled holes that were $4.00 \mathrm{~mm}$ in diameter, was placed above the ultrasonic water injector. (The perforated plate is important to the increase contact friction and ease vortex genesis). Furthermore, two axial fans (lower fan 1 and lower fan 2, from Fig. 1.) were also installed in the lower part of the chamber (part-A) to ensure a pressure potential inside the hollow box. The installed

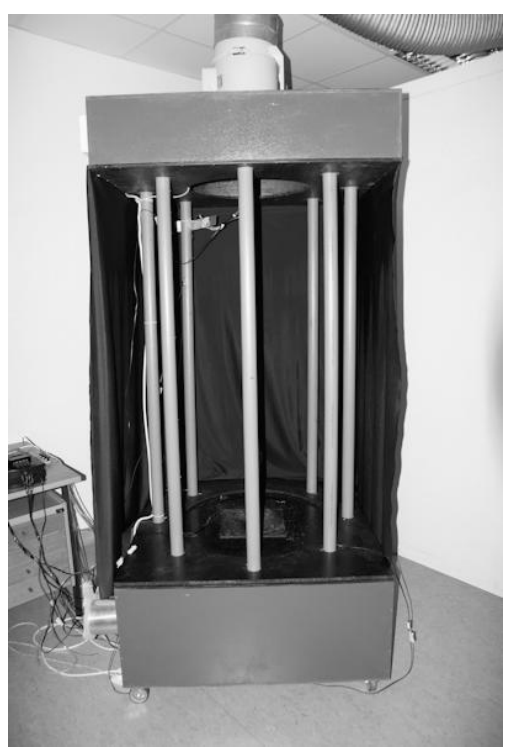

Fig. 1.b Simulation chamber

fans are important for the establishment of the radial inflow into the central part of the chamber (overall outlet air flow from the vertical tubes is equal to the flow ensured from the fan 1 and fan 2). Two fans are added to ensure the possibility of the airflow regulation. To provide varying flow conditions during the experimental work, an additional mechanical valve was installed on the suction side of the lower fan 1, Fig. 1. The middle of the simulation chamber, part-B, consists of eight vertical plastic pipes (tubes). The vertical tubes were drilled to establish radial airflow in the central region of the simulation chamber. At the bottom of the tubes (inside the hollow box A), larger diameter holes were drilled (compared to the diameter of the rest of the holes that were drilled in same vertical tubes).

The vertical tubes are adjustable, and they can be aligned to different angle positions, which ultimately affect the shape and the genesis of vortices (tube holes on the vertical tubes are oriented tangentially to the vortex axis). The last part of the simulation chamber is the upper part (part C, Fig. 1.), which is equipped with an axial vertical fan. The axial vertical fan is important for the establishment of the vertical updraft. Additionally, the vertical fan is equipped with a speed regulator, and the installed electrical power is approximately ten times larger than the installed power of the fans at the bottom part of the chamber (this ratio is important ratio for the genesis and maintenance of vortices). Below the vertical fan, a honeycomb filter was positioned to stabilise vortices, while a flexible hose was installed above the vertical fan to allow more precise measurements.

The simulation chamber, Fig. 1b, establishes vortices in a simple way by combining two separate airflows, i.e. a vertical updraft and a radial inflow. The ratio of the vertical and radial airflow masses is crucial for the genesis of vortices. Both previously mentioned airflows are artificially produced; according to the preliminary experimental results, the magnitude of the radial inflow is lower (airflow mass quantity) than that of the vertical 

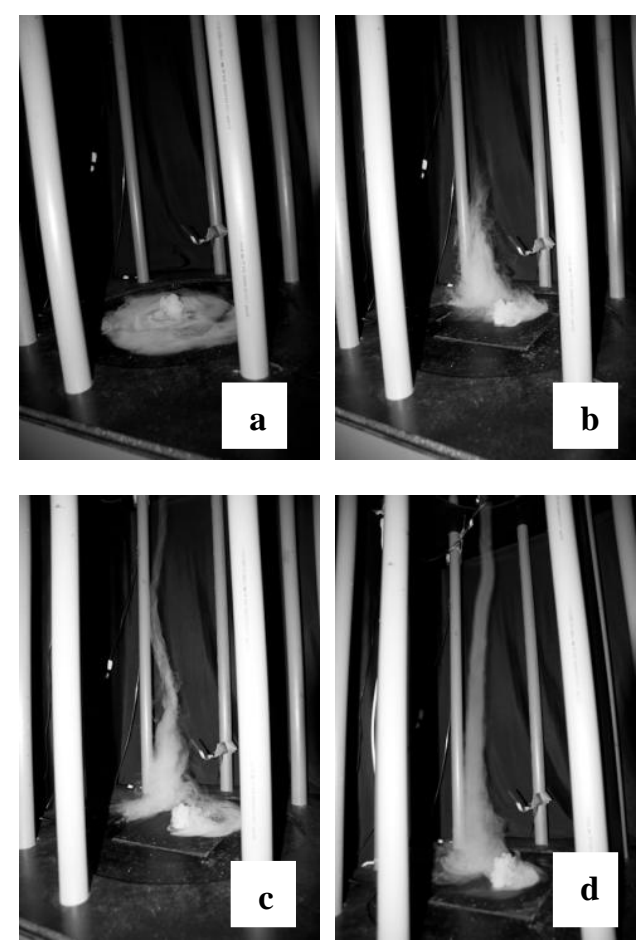

Fig. 2. Example of the characteristic vortex genesis in the developed simulation chamber

airflow. Several different shapes of vortices were successfully established, and Fig. 2 presents one characteristic example of vortex genesis (in which all characteristic phases of vortex genesis can be clearly noticed).

The measuring equipment used in this study consisted of two hot-wire anemometers, two sets of temperature/hygrometers and three pairs of differential manometers. Measuring equipment is installed at the bottom and at the top of the simulation chamber and around the region of the vertical tubes. Temperature and humidity of the surrounding air was also monitored. The average radial and tangential velocities have been used, regarding the vortex stability. Additionally, a vane anemometer was also used in some cases. The measuring range of the differential pressure sensors was +/- $500 \mathrm{~Pa}$ with a resolution of $0.017 \mathrm{~Pa}$. A data logger system was also used for the temperature/hygrometers and pressure sensors with appropriate software to enable data analysis. The used measuring equipment was modest due to limited funds. Therefore, the measurements were also restricted but still useful to derive conclusions. The preliminary experimental results will be discussed in detail in the remaining manuscript.

\section{PRELIMINARY EXPERIMENTAL RESULTS}

3.3 Influence of the water vapour content on the pressure potential

Three main parameters were observed and measured in the experimental analysis of the behaviour of convective vortices. The first measured parameter was

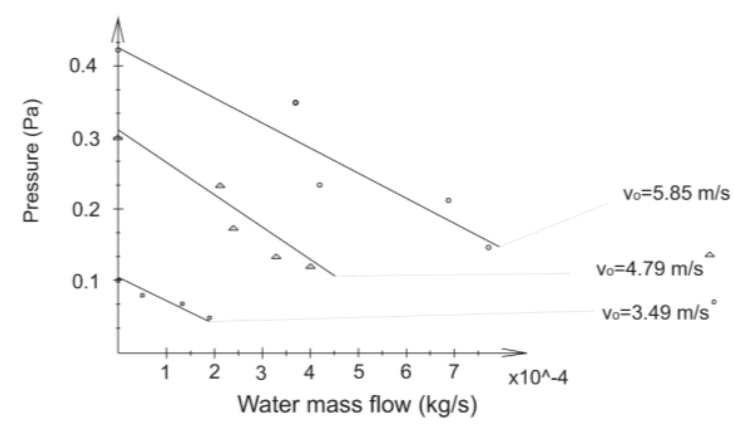

Fig. 3. Pressure drop potential as a function of the water vapour mass flow

the pressure drop in the vortex base, i.e., the pressure drop potential between the point in the central section of convective vortices and the surroundings (the point far away from the vortex influence). The second measured parameter was the magnitude of the radial inflow velocity, while the third measured parameter was the amount of vaporised water inside the vortex (i.e., the amount of water in the aerosol state) because the ultrasonic water injector was providing small dispersed water droplets. According to the provided measurement, the added water droplets had a loading effect on the vortex; they slowed the vortex and increased its intensity. In this case, a vortex expends more work to overcome the load produced by the addition of water, and this effect also affects vortex genesis (prolongs the time of vortices genesis). The previously mentioned effect was carefully visually observed in parallel with measurements. In addition to the measurement of the pressure drop potential of convective vortices, this study also attempted to measure and define the amount of work that is spent on air updraft and to overcome the friction losses in the vortex system.

As previously mentioned, the pressure potential was measured first by also including the influence of the added amount of the water in the vortex system. Furthermore, the amount of added water was measured with humidity measurement elements (sensors) that were installed in the flexible hose, Fig. 1. The amount of humidified water was indirectly measured based on available data for the overall mass flow and the absolute humidity perturbations in the vortex system. Furthermore, the change in the pressure drop was correlated with the change in the amount of water added to the vortex system. The results of these measurements are shown in the following figure, Fig. 3. Three series of measurements were purposely conducted to establish more precise results. Importantly, each measurement featured a different radial inflow velocity. The variation of the radial inflow velocity ensured that its influence on the vortex behaviour and stability could be observed. According to the provided measurement results, the vertical air mass flow remains the main influential parameter of the vortex intensity, irrespective of the intensity of the radial inflow velocity. In other words, a significant radial mass inflow, but not its velocity magnitude, is crucial to the formation of a strong vortex. This fact could also be proven for natural convective vortices, such as tornadoes, dust devils, etc. 
According to Fig. 3., the pressure drops were maximised when the air inflow radial velocities were maximised (from holes in the vertical tubes). Moreover, the first measurement (at an radial airflow velocity of $5.85 \mathrm{~m} / \mathrm{s}$ ) indicates that all added water vapour has had been aspirated in by the vortex system, irrespective of the change in the pressure drop. Therefore, the vortex remained stable even at the maximal amount of added water to the vortex system (the steamer maximum capacity was approximately $0.77 \mathrm{~g}_{\mathrm{w}} / \mathrm{s}$ ) and was not sensitive to the magnitude of the pressure difference. However, this fact was only true when the radial airflow velocity was maximised. For other, lower values of the radial airflow velocity, the quantity of added water is obviously physical limited, and the magnitude of the pressure potential is also restricted. For example, when the inflow radial velocity was reduced, as was the case in the second measurement with an air velocity of $4.79 \mathrm{~m} / \mathrm{s}$, the vortex could not aspirate the water. This phenomenon increased the humidity of the air at the bottom section of the vortex (the humidity was saturated at the bottom of the chamber, as shown in Fig. 2- picture a), which precluded the development of a vortex. In the previously mentioned circumstances, water condensation occurred at the bottom of the chamber as a direct consequence of the humidity saturation. Finally, the third measurement showed that the excess water content destroyed the vortex stability (in these circumstances, water vapour inflow in the air is near 0.2 $\mathrm{g}_{\mathrm{w}} / \mathrm{s}$ and the vortex disappears). The amount of water that can be added to the vortex system is obviously physically limited. The air saturates at a certain threshold of added water, beyond which additional water cannot be introduced to the system without physically destroying the vortex. Therefore, the pressure drop at the vortex base significantly decreases in these circumstances. Finally, the vortex system can withstand a certain amount of water without experiencing instability while experiencing some deceleration in vortex motion. As previously mentioned, the amount of water that can be added to the system appears to have a critical value, which needs to be determined. To this end, an analytical correlation between the added water content and vortex stability may be useful. However, the development of such an expression is expected to be difficult because each vortex system is unique in certain aspects.

\subsection{Specific friction work}

Natural convective vortices are similar to heat engines, in which the heat input from the surroundings is transformed into overall energy that is necessary for vortex genesis and its maintenance in the atmosphere. The crucial parameter that determines the magnitude of the convective vortex is the pressure potential. To this end, Rennó derived an equation that describes convective vortex systems. This equation generally relates the pressure difference to the specific energies (thermal-enthalpy change, kinetic and friction), Renno (2008),

$$
\Delta p \approx \rho_{s} \eta\left(c_{p} \Delta T_{n a}+l{ }_{v} \Delta q\right)+\rho_{s} \frac{\Delta v^{2}}{2}+\rho_{s} \eta W_{i r r}^{a b}
$$

Eq. (1) is only applicable when the specific factors $\gamma$ and $\eta$ can be determined (more detail regarding the derivation of eq. (1) can be found in Renno (2008). In this paper, the developed theoretical background by Rennó will be used to calculate these specific energies, i.e. to connect Renno's analytical approach to available experimental data and determine the specific friction work. Absolute values should be used to implement Renno's equation. However, absolute values could not be measured. Only the differences between energies in two specific states could be measured, and this approach enabled the determination of the specific friction work in an indirect way. Furthermore, the proposed research was conducted without the addition of heat into the vortex system. As such, Rennó's equation can be simplified and modified as follows for an adiabatically isolated system:

$\Delta(\Delta p)=\rho \cdot \Delta e_{k}+\rho \cdot \Delta e_{f}$

where $\Delta p$ represents the pressure drop (pressure potential), $\Delta(\Delta p)$ represents the pressure drop difference between two states, $\rho$ is the mean density of the air inside the vortex, $\Delta e_{k}$ is a specific change in the kinetic energy and $\Delta e_{f}$ is the specific change in the frictional energy. According to the preliminary measurement results, an increase in the water content in the simulation chamber decreases the kinetic energy of the vortex system (as concluded in the previous section due to the additional work that is needed to lift the added water vapour). However, if significantly more water is pulled into the vortex system, the kinetic energy will increase due to the overall increase in the air mass flow. Because an increase in the water mass is not measurable, it can be neglected. Conversely, a change in the kinetic energy (caused by the added water amount in the vortex system) should be taken into account, but only when a significant amount of water can be pulled into the vortex system. The overall change in the kinetic energy between the two series of measurement can be expressed with the following equation

$\Delta e_{k}=\frac{\dot{m_{1, w}}}{\dot{m_{1, a l l}}} \frac{v_{1}^{2}}{2}+\left(\frac{v_{1}^{2}}{2}-\frac{v_{0}^{2}}{2}\right)$

where $\Delta e_{k}$ denotes the difference in the kinetic energy between two measurement series (labelled series 0 and 1), $\dot{m}_{1, w}$ is the difference in the value of vaporised water mass flow between two measurement series, $\dot{m}_{1, a l l}$ is the overall mass flow $v_{1}$ and $v_{0}$ represent the measured radial inflow velocities of vortex system for the measurement series. The change in the friction energy was calculated using the two previous equations (eq. (2) and eq.(3)) and the available measured results. Namely, the pressure difference, $\Delta(\Delta p)$, and $\Delta e_{k}$ were measured, and $\Delta e_{f}$ was calculated from eq. (2). The specific measured results are presented in Fig. 4, in which the specific friction work, i.e., its change between two measurement series, is shown as a function of the water mass flow in the vortex system. Fig. 4 indicates that an increase in the radial air velocity decreased the specific friction work. This effect was 


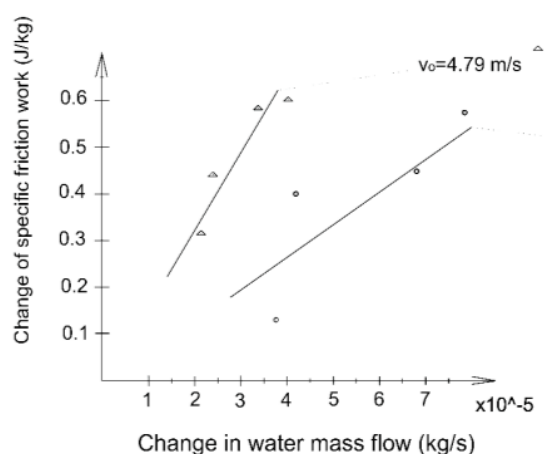

Fig. 4. Specific friction work change as a function of the water vapour mass flow

attributed to increased air flow intake into the vortex system (i.e., the contact friction area is increased in this case, which decreases the specific friction work, as it is distributed on a larger surface).

The overall change in the specific work (which includes work that is transformed into kinetic energy and friction work that occurs in the vortex system) is also interesting to observe, as shown in Fig. 5. Namely, the specific work positively correlates with the amount of water added to the system to ensure the stability of the vortex, which increases its kinetic energy. In this situation, the vortex system needs to increase the specific work to lift the intake of added water and simultaneously ensure vortex stability. The higher values of the radial airflow velocities enable the addition of more water to the vortex system without compromising the stability of the vortex.

\subsection{Specific vortex efficiency}

The vortex was mechanically simulated in the experimental approach proposed in this paper, i.e., heat was not added to the convective vortex system and only the pure mechanical aspect was analysed. Therefore, a new factor can be introduced, i.e., the specific vortex efficiency factor, which connects the produced mechanical work with the overall work spent in the vortex system. This factor aims to elucidate the amount of produced work that could be extracted from the vortex system in form of the useful mechanical work without physically destroying it. Hence, the specific vortex efficiency can be defined as the ratio of the amount of the produced work to the amount of the overall energy that is spent in the vortex system (kinetic work and friction work on the vaporised vortex system). Hence, the specific vortex efficiency can generally be defined as follows:

$$
\varepsilon=\frac{\Delta w_{v}}{\Delta w_{v}+\Delta e_{f}}
$$

where $\Delta w_{v}$ is a change in the produced work between two measurement series. According to the provided measurements, the maximum specific efficiency of the mechanically generated vortex (without heat influence) was calculated to be approximately $0.8 \%$ on average. Furthermore, the specific vortex efficiency was

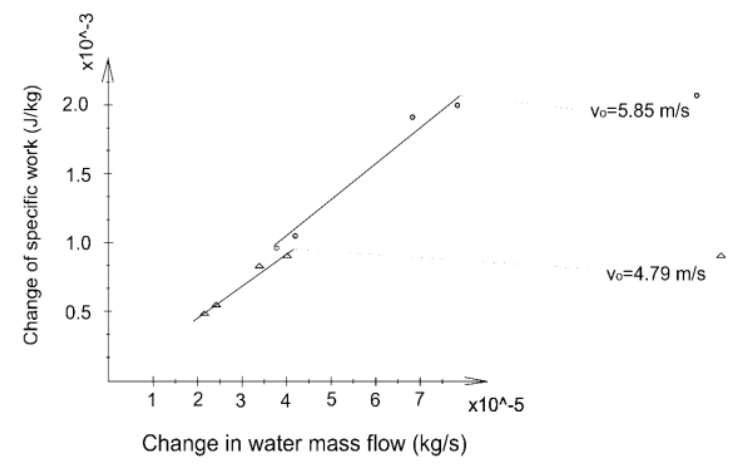

Fig. 5. Change in specific work as a function of the water vapour mass flow

maximised for higher values of the air mass flow rates, which indicates that the air mass flow intake into the vortex system is the most influential parameter. Namely, the specific efficiency positively correlates with the radial mass flow, while the radial velocity also improves the specific work efficiency, but to a much smaller degree. As a consequence, the radial velocity positively correlates with the friction work, while the mass flow positively correlates with the mass of fluid available for work in vortex system. Furthermore, the magnitude of the radial air mass inflow of convective vortices is directly connected to the heat input. However, as previously mentioned in this paper, the influence of heat was not analysed; only the mechanical influence was examined in this study. The amount of equivalent heat necessary to increase the specific vortex efficiency of the system would be interesting to analyse. The value of the specific vortex efficiency positively correlates with the amount of available mechanical work that could be used to generate electricity, for example. This concept is elaborated in previous studies (Michaud $(1975,1999)$ and Ninic and Nizetic (2009)) which utilised convective vortices as heat engines.

\section{CONCLUSIONS}

This paper presents a specific experimental setup that could simulate vortices in different circumstances. The influence of the heat input was not analysed in this research, but the developed chamber can be easily set up to include the heat influence for such studies. Although Grazulis provided the original simulation design, several modifications were implemented in this study to expand the chamber facilities and ensure flexibility. The specific simulation chamber features have also been elaborated in detail and the working principle was described. Furthermore, artificial vortices were repeatedly and successfully created in the developed simulation chamber, and one specific case of vortex genesis was presented. According to the preliminary experimental measurements, an increase in the water content in the vortex system decreases the pressure potential and weakens the vortex structure when the magnitude of the radial inflow velocity is constant (because more available work is spent on lifting the added water). The specific vortex efficiency factor was introduced as a novelty during the mechanical analysis in this paper. A low magnitude of the vortex efficiency factor can be expected when heat 
is not added to the vortex system, which proves that a certain quantity of the specific work can still be extracted from the vortex system without a loss of vortex stability, even when heat is not added to the vortex system. However, a significant heat input in the vortex system must be ensured if a significant amount of mechanical work is extracted from the system (like when convective vortices are used as heat engines). In this case, the specific vortex efficiency can be increased, which indicates more useful mechanical work for electricity production. Finally, this paper specifically focused on the experimental setup, and further research work will be aimed at the detailed experimental investigation of parameters that influence the genesis and stability of vortices, such as when heat is added to the vortex system. Such studies require a serious investment in the measuring equipment, which should be ensured prior to continuing research on this topic.

\section{ACKNOWLEDGEMENTS}

The authors would like to thank the Croatian Ministry of Science, Education and Sports (research project 0230231751-3011) and the Carlsberg Croatia d.o.o. for the funding of this project.

\section{REFERENCES}

Bech J., Gayà M., Aran M., Figuerola F., Amaro J., Arús J. (2009): Tornado damage analysis of a forest area using site survey observations, radar data and a simple analytical vortex model, Atmospheric Research 93(1-3),118-130.

Bluestein H.B., Pazmany A.L.(2000): Observations of tornadoes and other convectivephenomena with a mobile, 3.mm wavelength, Doppler radar: The spring 1999 field experiment, Bull.Am. Meteorol. Soc. 81, 2939-2951.

Chandrasekar V., Wang Y. , Bharadwaj N., Zhang S., Martinez M., Mclaughlin D., Brotzge J., Phillips B. (2010): Observation of an EF2 tornado winds using networked radar test-bed, IEEE National Radar Conference - Proceedings, art. no. 5494443, 1167-1171.

Dessens J. (1962): Man-Made tornadoes. Nature 193, 13-14.

Firouzabadi B., Afshin H., Bagherpour A.(2010). Experimental investigation of trubulence specifications of turbidity currents. Journal of applied fluid mechanics 3(1), 63-73

Emanuel K.A. (1989): An air-sea interaction theory for tropical cyclones. Part I: Steady-state maintenance. J. Atmos. Sci. 43, 585-604.

Fred L., Haan J.R., Partha P., Sarkar W. , Gallus A. (2008): Design, construction and performance of a large tornado simulator for wind engineering applications, Engineering Structures 30 (4), 1146-1159.
Grazulis, T.P. (1998):A demonstration of vortex coinfigurations in an expensive tornado simulator, 19th Conf. Severe Local Storms, Minneapolis, MN Amer. Meteor. Soc., 85-88.

Hasegawa H., Kumagai, S. (2008). Adaptive separation control system using vortex generator jets for timevarying flow. Journal of applied fluid mechanics $1(2), 9-16$

Hess G.D., Spillane K.T. (1990): Characteristic of dust devils in Australia, J. Appl. Meteorol 27, 305-317.

Inoue H.Y., Kusunoki K., Kato W., Suzuki H., Imai T., Takemi T., Bessho K., Suzuki O. (2011) Finescale doppler radar observation of a tornado and lowlevel misocyclones within a winter storm inthe Japan Sea coastal region, Monthly Weather Review 139 (2), 351-369.

Mattsson J.O., Nihlen T., Yue W.(1993):Observations of dust devils in a semi-arid district of southern Tunisia, Weather 48, 359-363.

Metzger S., Balme M.R., Farell W.M, Fuerstenau S., Greeley R., Merrison J., Patel M.R., Ringrose T.J., Towner M.C., Zarnecki J.C. (2002): Unwrapping the whirlwind: Measuring natural dust devils, Geol. Soc. Am. Abstr. Programs 36, 5-11.

Michaud L.M. (1975): Proposal for the use if a controlled Tornado-like Vortex to Capture the Mechanical Energy Produced in the Atmosphere from Solar Energy. Bulletin American Meteorological Society 56(5),530-534.

Michaud L.M. (1995): Heat to work conversion during upward heat convection, Part I: Carnot engine method. Atmospheric research 39,157-178.

Michaud L.M.(1999):Vortex process for capturing mechanical energy during upward heat- convection in the atmosphere. Applied Energy 62(4), 241-251.

Michaud L.M.(1996): Heat to work conversion during upward heat convection, Part II: Internally generated entropy method. Atmospheric research $41,93-108$.

Michaud L.M.(2013). Web source: http://www.vortexengine.ca/

Mukunda, P.G., Shailesh R.A., Kiran, A.S., Shrikantha S.R.(2009). Experimental studies of flow patterns of different fluids in a partially rotating cylinder. Journal of applied fluid mechanics 2(1), 39-43.

Natarajan D., Hangan H. (2012): Large eddy simulations of translation and surface roughness effects on tornado-like vortices, J. Wind EnG. Ind. Aerodyn Vol.104-106, 577-584.

Ninic N., Juric Z., Nizetic S.(2006): Thermodynamical aspect of definitions "CAPE" and "TCAPE'. Geofizika 23(2), 143-154. 
Ninic N., Nizetic S. (2009): Elementary theory of stationary vortex columns for solar chimney power plants. Solar Energy 83(4), 462-476.

Nizetic S. (2010):An atmospheric gravitational vortex as a flow object: improvement of the three-layer model, Geofizika 27(1),1-20.

Nizetic S.(2011): Technical utilisation of convective vortices for carbon-free electricity production: A review. Energy 36, 1236-1242.

Palmer R.D., Bodine D., Kumjian M., Cheong B., Zhang G., Cao Q., Bluestein H.B., Wang Y. (2011): Observations of the 10 may 2010 tornado outbreak using OU-PRIME: Potential for new science with high-resolution polarimetric radar, Bulletin of the American Meteorological Society 92 (7), 871-891.

Paul A.R., Kuppa K., Yaday M.S., Dutta U. (2011). Flow improvement in rectangular air intake by submerged vortex generators. Journal of the applied fluid dynamics 4(2), 77-86.

Renno N., Ingersoll A.P. (1996): Natural convection as a heat engine: a theory for CAPE. Journal of the Atmospheric Sciences 53, 572-585.

Renno N.O., Burkett M.L., Larkin M.P.(1998): A simple theory for dust devils. J.Atmos. Sci. 55, 3211-3252.
Renno N.O., Bluestein H.B.(2001) A simple theory for waterspouts, J. Atmos. Sci. 58, (2001), 927-932.

Renno N.O. (2004): Matador 2002: A pilot field experiment on convective plumes and dust devils, J. Atmos. Sci 55, 3224-3252.

Renno N. (2008): A thermodynamically general theory for convective vortices. Tellus 60A, 688-699.

Sobolik V., Jirout T., Havlica J., Kristiawan N. (2011). Wallshear stress in taylor vortex flow. Journal of applied fluid mechanics 4(2), 25-31.

Tari P.H., Roi G., Hangan H. (2010): Experimental investigation of tornado-like vortex dynamics with swirl ratio: The mean and turbulent flow fields, Journal of Wind Engineering and Industrial Aerodynamics Vol. 98 (12), 936-944.

Wurman J., Kosiba K., Robinson P. (2013): In situ, Doppler radar, and video observations of the interior structure of a tornado and the winddamage relationship, Bulletin of the American Meteorological Society 94 (6), 835-846. 